\title{
EDITORIAL
}

\section{Less talk, more action}

\author{
The publication of the second volume of England's annual health report has increased \\ pressure to prioritize strategies to tackle the antibiotic resistance crisis.
}

The deployment of antibiotics for the treatment of infectious diseases was arguably the biggest medical success story of the twentieth century. However, both the overuse and misuse of antibiotics in human and veterinary medicine and animal husbandry have hastened a global resistance crisis that, in the future, could see a surge in infection-related mortality. Although microbiologists have warned of the threat for years, what has been lacking is clear leadership to tackle the problem. However, in the United Kingdom, momentum now seems to be gathering following the recent publication of the second volume of the Chief Medical Officer's annual health report, which details the current antibiotic resistance burden in England and, more importantly, provides a list of recommendations to manage the crisis.

Bacterial resistance to antibiotics is by no means new, and readers will doubtless be familiar with reports of the spread of so-called superbugs in recent years. Although improved hospital infection control measures have led to a decline in the incidence of methicillin-resistant Staphylococcus aureus infection in the United Kingdom since its peak in 2003, a new wave of multidrug-resistant Gram-negative bacteria, most notably carbapenemresistant Enterobacteriaceae family members, are becoming a major cause for concern, not only in the United Kingdom but also in the United States. In addition, the emergence of extensively resistant Mycobacterium tuberculosis and the rise of Neisseria gonorrhoeae resistance means that we have very few, if any, drugs available for the treatment of these infections. Altogether, the data paint a very disturbing picture - the more we use antibiotics, the more we lose them; however, if we refrain from using them, we cannot capitalize on their benefits.

The current crisis is compounded by a litany of issues, including a lack of awareness of the problem outside the microbiology and medical fields, a limited understanding of the factors that lead to resistance, insufficient surveillance data, inadequate reporting and sharing of data both nationally and internationally, increased mobility of populations, the inappropriate use of available antibiotics, and the failure of the drug development industry to bring new antibiotics to market. For pharmaceutical companies, the lack of return on investment means that they are not incentivized to develop novel classes of antimicrobials, and this had led to proposals for a re-evaluation of pharmaceutical business models.
Against this background, the report from Dame Sally Davies sets out 17 recommendations to tackle the current threat. Of particular note is the call to put antibiotic resistance on the UK national risk register for civil emergencies. A civil emergency is defined as "an event or situation which threatens serious damage to human welfare", and the register currently recognizes pandemic influenza as the highest priority risk. The report also encourages health care professionals and policy makers to work together to ensure the prudent and sparing use of currently available antimicrobials, as well as to raise awareness and promote change in health care practices, including more stringent antibiotic stewardship. Academics are also advised to collaborate with national health organizations to improve the accessibility of surveillance data in order to build a better picture of the problem on both a national and global scale. Moreover, politicians are encouraged to address the global nature of the problem by strengthening international collaborations to ensure the development of new drugs.

The timing of the report reflects the precipitous nature of the crisis. Given the scale and urgency of the problem, funding for both basic and applied research into antibiotic resistance should be a priority. It was therefore disappointing to see, in a recent analysis of the investments awarded to UK institutions for infectious disease research in 1997-2010, that only 3.7\% ( $£ 96$ million) of the $£ 2.6$ billion total was devoted to research on antimicrobial resistance ${ }^{1}$. This is reflected in informal feedback we have received from UK microbiologists, who are concerned about the drop in extramural funding for bacteriology; for example, it is estimated that funding for bacteriology from the UK Medical Research council has been reduced from $\sim 9 \%$ of the total budget in 2009 to $\sim 3 \%$ in 2011. Moreover, despite years of effort from the community, there is still no UK national research centre dedicated to this problem.

We cannot eradicate resistance. It is therefore imperative that we get to grips with how to manage the current crisis. Implementation of the recommendations in Davies' report will require strong political leadership and an integrated effort from all parties concerned. It is time for less talk and more action if we are to avoid a return to the pre-antibiotic era.

1. Head, M. G. et al. Lancet Infect. Dis. 13, 55-64 (2013). 